The vast repository of evidence recorded geologically over the millennia is the best place to find these floods.

Knox deliberately chose to study the flood-prone Upper Mississippi, because it drains a large area and because it lies at the confluence of two climate systems, making it particularly sensitive to climate variations. Now that we see that the approach works there, we should try looking in the tropics where monsoons, storms and El Niño/Southern Oscillation climate fluctuations may be particularly sensitive to greenhouse warming.

Vic Baker is in the Department of Geosciences, University of Arizona, Tucson, Arizona 85721, USA.

\title{
MOLECULAR EVOLUTION
}

\section{Genes-in-pieces revisited}

\section{W. Ford Doolittle and Arlin Stoltzfus}

ON page 470 of this issue ${ }^{1}$, Tittiger et al. describe an intron in the triosephosphate isomerase (TIM or TPI) gene of the mosquito Culex tarsalis, an intron that neatly subdivides an exon that would otherwise encode two protein 'modules'. This is exciting because it confirms a prediction by Gilbert et al. ${ }^{2}$, and may bring joy to supporters of the introns early' theory of gene evolution.

First some history. Immediately upon the discovery of introns in 1977, Walter Gilbert proposed in these pages ${ }^{3}$ that the split gene organization of eukaryotes serves evolution. By separating proteincoding information into many little bits, he ventured, introns facilitate genetic rearrangements which offer up novel mosaic genes to the scrutiny of natural selection. For some of the recently assembled genes of the vertebrate bloodclotting system, Gilbert has been proved right - mosaic evolution respecting exon boundaries is observed ${ }^{4}$

A bit later, Darnell ${ }^{5}$ and one of us ${ }^{6}$, reasoning that the long-term advantage conferred by the ability to shuffle exons could not itself account for the existence of introns, ventured that these intervening sequences were really evolutionary relics. In this 'introns early' view, spliceosomal introns mark the positions at which ancient (and independently replicating) exons were joined to form the first modern-sized genes. Their absence from present-day prokaryotes could be due to sudden or gradual deletion over the past three billion years - 'streamlining'.

Another way to explain split genes ('introns late') has it that spliceosomal introns are descendants of transposable elements which invaded the eukaryotic lineage sometime after its divergence from prokaryotes. Some spliceosomal introns do indeed seem to have arisen recently and some introns do contain mobility-conferring genes ${ }^{7}$. However, proving each and every contemporary intron a relic is not the point. The real question is: are at least some modern exons the descendants of small, separately evolved units from which the first genes were assembled?

Two different sorts of data seem relevant. The first, surveys of the phylogenetic distribution of individual introns and the spliceosomal machinery, are not friendly to 'introns early'. Introns in similar positions of homologous genes (TPI genes, for instance) from animals, plants and fungi are almost irrelevant. There are very many independent evolutionary divergences in eukaryotes much deeper than these, and still many below that leading to euglenoids, the most deeply diverged lineage known to have introns ${ }^{8}$. Lineages further from us than euglenoids may lack introns and the spliceosomal machinery. If so, we must imagine many independent instances in which eukaryotic (as well as prokaryotic) genomes would have had to be streamlined - possible, but very unparsimonious $^{7,9}$. (Group I introns found in eubacteria have no bearing on the situation, in the absence of any other reason to believe them ancestral to spliceosomal introns.)

The second sort of relevant data harkens back to Blake's 1978 suggestion $^{10}$ that jumbling protein pieces together at random, without regard to structural motifs and functions, would produce only garbage. Blake's original view that exons should encode domains has been revised, and now advocates of the introns-early theory seek a variety of correlations at lower levels of protein structure $^{11}$. Indeed, believable correlations between gene structure and protein structure can be seen for some genes recently produced by shuffling - like those of the blood-clotting system ${ }^{4}$.

But again, this limited data set says nothing about the ancestral situation. Repeated shuffling selects for exons that mix well together, even if they were created initially by insertion of intron/ transposons at positions that were random with respect to protein structure. So Gilbert et al. ${ }^{2}$ proposed that we focus on ancient genes, produced before the earliest evolutionary divergence (that between eubacteria and eukaryotes). If such genes boast exons that encode dis- crete units of structure or function at the protein level, those exons must have had previous evolutionary history as discrete units, possibly even before the first cells.

TPI has been one of the three or four favourite ancient proteins. Gilbert et $a l^{2}$, after examining the structures of TPI genes from chicken, maize and Aspergillus, deduced that the ancestral form had eleven exons, each encoding a discrete unit of structural compactness, as defined by $\mathrm{Go}^{11}$. Nine of the original ten introns were found in at least one modern gene. The missing tenth intron would have divided the third modern exon in two, and Gilbert et al. ventured to "predict that in some other [TPI] gene there will be an intron that breaks up this exon". It is this prediction that Tittiger et al. have made real.

So does this save the day for introns early? It depends on how sympathetically one reads ref. 2 . The null expectation of failure was as low as zero (as forbidden intron positions were not specified), or as high as 92 per cent (as the context suggests that nearly all possible positions can be viewed as forbidden - a strict interpretation implying that, in future, no new intron positions are expected). These odds are more compelling than the previous detailed analysis of TPI by Go and Nosaka ${ }^{12}$ : with the broad definition of module boundaries allowed by these authors, most randomly placed introns would have been taken as consistent with introns early.

Prudence calls for a more rigorous analysis of many more of the data. Ideas about what are the relevant features of protein structure can be too easily modified to fit the gene structure data better. Analytical rules that have evolved in the analysis of TPI should therefore be applied blindly to all other proteins for which there are adequate structural and genetic data. To our knowledge, the essential step of excluding the null hypothesis (of randomly placed introns) has not been taken, although there are surely now enough data to attempt this.

W. Ford Doolittle and Arlin Stoltzfus are in the Canadian Institute for Advanced Research, Department of Biochemistry, Dalhousie University, Halifax, Nova Scotia B3H 4 H7, Canada.

1. Tittiger, C., Whyard, S. \& Walker, V. K. Nature 361 470-472 (1993).

Gilbert, W. et al. Cell 46, 151-154 (1986)

3. Gilbert, W. Nature 271, 501 (1978).

4. Blake, C. C. F., Harlos, K. \& Holland, S. K. Cold Spring Harbor Symp. quant. Biol. 52, 925-931 (1987).

5. Darnell, J. E., Jr Science 202, 1257-1260 (1978)

6. Doolittle, W. F. Nature 272, 581-582 (1978)

7. Cavalier-Smith, T. Trends Genet. 7, 145-149 (1991)

8. Muchal, U. S. \& Schwartzbach, S. D. PI. molec. Biol. 18, 287-299 (1992)

. Palmer, J. D. \& Logsdon, J. M., Jr Curr. Opinion Genet. Dev. 1, 470-477 (1991).

10. Blake, C. C. F. Nature 273, 267-268 (1978).

11. Go, M. Nature 291, 90-92 (1981).

12. Go, M. \& Nosaka, M. Cold Spring Harbor Symp. quant Biol. 52, 915-924 (1987). 